\title{
Recognition and tethering of transport vesicles at the Golgi apparatus
}

DOI:

10.1016/j.ceb.2017.02.003

Document Version

Accepted author manuscript

Link to publication record in Manchester Research Explorer

\section{Citation for published version (APA):}

Witkos, T., \& Lowe, M. (2017). Recognition and tethering of transport vesicles at the Golgi apparatus. Current opinion in cell biology, 47, 16-23. https://doi.org/10.1016/j.ceb.2017.02.003

\section{Published in:}

Current opinion in cell biology

\section{Citing this paper}

Please note that where the full-text provided on Manchester Research Explorer is the Author Accepted Manuscript or Proof version this may differ from the final Published version. If citing, it is advised that you check and use the publisher's definitive version.

\section{General rights}

Copyright and moral rights for the publications made accessible in the Research Explorer are retained by the authors and/or other copyright owners and it is a condition of accessing publications that users recognise and abide by the legal requirements associated with these rights.

\section{Takedown policy}

If you believe that this document breaches copyright please refer to the University of Manchester's Takedown Procedures [http://man.ac.uk/04Y6Bo] or contact uml.scholarlycommunications@manchester.ac.uk providing relevant details, so we can investigate your claim.

\section{OPEN ACCESS}




\section{Recognition and tethering of transport vesicles at the Golgi apparatus}

Tomasz M. Witkos and Martin Lowe

Faculty of Biology, Medicine and Health, University of Manchester, Michael Smith

Building, Oxford Road, Manchester, M13 9PT, UK

Email: martin.lowe@manchester.ac.uk

Short title: Vesicle recognition and tethering at the Golgi apparatus

Word count: 3,831 (excluding references and figure legends) 


\begin{abstract}
The Golgi apparatus occupies a central position within the secretory pathway where it is a hub for vesicle trafficking. Distinct classes of transport vesicles traffic diverse cargoes into and out of this organelle, as well as between the different Golgi sub-compartments. A key feature of Golgi trafficking is the specific recognition of transport vesicles at the different regions of the Golgi apparatus, required for the correct cargo delivery. Specificity is ensured by coiled-coil golgins and multi-subunit tethering complexes (MTCs), which act together to capture vesicles and promote their subsequent fusion with the Golgi membrane. In this review we discuss our current understanding of how golgins and MTCs function together to mediate the specific recognition of vesicles at the Golgi apparatus.
\end{abstract}




\section{Introduction}

The endomembrane system is a complex and highly dynamic network of membranebound compartments through which a multitude of diverse cargo molecules are trafficked. In most cases cargo is moved between the compartments in transport vesicles, which bud from one compartment and fuse with the next compartment in the pathway. It is vital that cargo is delivered to the correct destination, not only for the efficacy of trafficking per se, but also to ensure that organization of the endomembrane system is maintained. Specificity is ensured by a number of molecular machineries that mediate both targeting and fusion of vesicles at the correct downstream compartment. These machineries act in concert to capture the transport vesicle, a process referred to as tethering, which is followed by docking, the close apposition of vesicle and target membranes, and finally fusion, upon which vesicle cargo is delivered. In this review we will discuss our current understanding of vesicle recognition at the Golgi apparatus, an organelle that lies at the heart of the endomembrane system.

The Golgi apparatus is comprised of distinct compartments called cisternae that are layered on top of one another to form the Golgi stack [1]. In certain eukaryotes the Golgi stacks exist as discrete entities, with each cell having one or more stacks, while in most vertebrate cells hundreds of Golgi stacks are laterally connected to form the Golgi ribbon, which is usually positioned next to the centrosome [1]. Newly synthesized secretory cargo is delivered from the endoplasmic reticulum (ER) into the cis-side of the Golgi apparatus [2]. Retrograde carriers recycle escaped ER residents and misfolded proteins, certain signaling molecules, as well as those membrane proteins that constitute trafficking machinery, back to the ER, while secretory cargo is trafficked onwards 
through the Golgi stack [3]. Such cargo is transported vectorially across the stack, providing access to resident enzymes within the Golgi cisternae that sequentially modify the cargo, most notably at the level of glycan processing [4]. Several models have been proposed for intra-Golgi trafficking, with cisternal maturation favored for most cargoes [5]. In this model cargo is retained within cisternae that progressively mature as they migrate across the Golgi stack, a process driven by vesicle-mediated recycling of Golgi resident proteins from later to earlier cisternae that results in a progressive change to cisternal composition and identity [6,7]. Following transition across the Golgi stack, secretory cargo is sorted and packaged into trafficking intermediates at the trans-Golgi network (TGN) for delivery to various post-Golgi compartments, which include the plasma membrane, secretory vesicles, and endosomes [8]. In addition to sorting cargo for exit from the Golgi, the TGN also receives incoming material that is trafficked mostly from endosomal compartments [9]. Given its central position within the secretory pathway, and the fact that it interfaces with the endosomal system, with numerous incoming and outgoing trafficking intermediates, the Golgi apparatus can be viewed as a hub for membrane traffic. Because of the complexity of trafficking at the Golgi, it is imperative that transport vesicles are recognized in a highly specific manner at the various distinct regions of this organelle. Specificity is provided by vesicle tethering proteins and SNAREs, which act in a coordinated and likely sequential manner to facilitate vesicle capture at the Golgi membrane followed by the subsequent steps that terminate in vesicle fusion (Figure 1).

\section{Vesicle capture by golgins}


The initial capture or tethering of transport vesicles at the Golgi apparatus is most likely mediated by golgins, a family of elongated coiled-coil tethering proteins, of which there are at least eleven members $[10,11]$. Golgins are anchored to the Golgi membrane via their carboxy terminus, either through binding to membrane-associated small GTPases of the Arf, Arl or Rab families, or through a membrane integrated tail anchor $[10,11]$. Due to their coiled-coil nature, golgins are thought extend a considerable distance (predicted to be in the range of $100-600 \mathrm{~nm}$ ) into the surrounding cytoplasm. This organization is ideal for capturing vesicles at long range, and as such is likely to increase the efficiency of trafficking. The golgins also play a major role in dictating specificity of trafficking at the Golgi apparatus. Using an elegant gain-of-function assay in which golgins were targeted to mitochondria, Wong and Munro were able to show, firstly, that golgins are sufficient to tether transport vesicles, and secondly, that different golgins tether different types of vesicles [12**]. For example, TGN golgins selectively captured endosomederived vesicles, whereas those at the cis-Golgi captured vesicles bringing cargo from the ER, and golgins at the cisternal rims tethered intra-Golgi transport vesicles. The ability of different golgins to tether different vesicles therefore tallies with their differential localization to distinct Golgi regions (Figure 2).

\section{Tethering motifs}

For optimum efficiency of vesicle capture by golgins we may predict that they tether vesicles at sites distal to the membrane; that is by their amino terminal region. For most golgins this appears to be the case. The best understood example is GMAP-210, which tethers transport vesicles via an amino terminal amphipathic lipid-packing sensor (ALPS) motif $\left[13,14^{*}\right]$. The ALPS motif binds to membrane vesicles based upon their size and 
their lipid composition and packing [15*]. Surprisingly, these properties alone are sufficient for the selective capture of vesicles by GMAP-210 at the cis-Golgi, leading to the proposal that GMAP-210 acts as a vesicle selectivity filter at the cis-Golgi [15*]. Other golgins lack an ALPS motif and use a different mechanism to capture vesicles. GM130 binds the vesicle docking protein p115 via its membrane-distal amino terminal region [16], and it has been proposed that p115 interaction supports vesicle tethering during secretory traffic and post-mitotic Golgi assembly [16-18]. However, GM130 lacking the p115 binding site is able to tether transport vesicles when rerouted to mitochondria $\left[12^{* *}\right]$, so it is likely other determinants can also support tethering by GM130. It is therefore possible that GM130 has two tethering motifs, one that can bind p115 and another that remains to be identified.

The vesicle tethering motifs of several other golgins have recently been identified $\left[19^{* *}\right]$. These motifs are located at the extreme amino terminus, and act in a selective manner to tether vesicles. Tethering of intra-Golgi vesicles is mediated by a conserved motif found in golgin-84, TMF 1, and GMAP-210. In the latter case, the newly identified tethering motif is distinct from the ALPS motif, and, interestingly, it appears to capture vesicles that are distinct from those captured by the ALPS motif, indicating that GMAP210 can tether different vesicles using two separate mechanisms. Tethering of endosomederived vesicles by the TGN golgins is mediated by motifs that are distinct from those that capture vesicles earlier in the Golgi [19**]. Interestingly, GCC88 uses a motif that is different to that found in both golgin-97 and golgin-245, suggesting it tethers different endosome-derived carriers to those tethered by the other two golgins [19**]. Although the tethering motif of the TGN golgin GCC185 is unknown, in vitro experiments have 
indicated that it can tether transport vesicles through its amino terminal region, whose sequence differs from that of other TGN golgins [20**]. Structural analysis has indicated that this region can adopt a splayed conformation, which may allow increased avidity of binding through 'cradling' of the spherical vesicle by the two splayed ends of the protein $\left[20^{* *}\right]$.

\section{Golgins and Rab GTPases: getting closer to the membrane}

All known golgins bind to Rab GTPases, raising the formal possibility that vesicle capture could occur through interactions between the golgins and vesicle-associated Rabs. However, Rab binding sites are distinct from the amino terminal tethering motifs described above, and tend to be located along the length of golgins [21], which argues against a role in vesicle capture. Therefore, Rab binding is likely more important for the steps that follow capture, where the vesicle has to move closer to the target membrane to undergo docking and fusion (Figure 1). In the case of GMAP-210, it has been shown that Rab2 binding does not contribute to the initial tethering event, but is required for full functionality of GMAP-210 at the Golgi apparatus, supporting the idea that Rab2 binding acts downstream of tethering [14*]. Interaction between the vesicle-bound Rab and Golgi-membrane associated golgins could allow the vesicle to hop down the length of a golgin, or even onto an adjacent golgin able to bind the same Rab, helping it navigate its way through the meshwork of golgin 'tentacles' to contact the Golgi membrane [22]. Another scenario is that Rab binding may induce a conformational change in the golgin, such that the vesicle is brought into close proximity to the Golgi membrane, which may occur by bending of the golgin, or via its collapse. The latter has been demonstrated for the endosomal coiled-coil tethering protein EEA1, which undergoes entropic collapse 
upon binding to Rab5 [23*]. Golgins have numerous breaks in their coiled-coil domains, implying a high degree of flexibility, and, at least for GCC185 and GM130, it has been shown that they can fold into shorter conformations through bending at internal flexible hinge regions $[20 * *, 24]$.

\section{Multi-subunit tethering complexes -orchestrators of tethering downstream from golgins}

Following vesicle capture by golgins and Rab engagement additional interactions are required to mediate the transition from tethering to docking and fusion (Figure 1). This is where the multi-subunit tethering complexes (MTCs) most likely function. The MTCs are found at various locations within the endomembrane system, and as the name suggests they comprise multiple subunits of varying size [25]. Three of the MTCs have been localized to the Golgi apparatus, namely Transport Protein Particle (TRAPP), Conserved Oligomeric Golgi (COG), and Golgi-associated Retrograde Protein (GARP). There are three TRAPP complexes, designated TRAPPI-III, with the core TRAPPI complex localized to the cis-Golgi, and TRAPII and TRAPIII, formed by the association of additional subunits, located later in the secretory pathway [26,27]. The TRAPP complexes were initially through to function as vesicle tethers, but their activity in tethering is likely indirect. The ability of TRAPP to function as a guanine nucleotide exchange factor (GEF) for Rab1 (Ypt1 in yeast) at the cis-Golgi [28-30], and Rab11 (Ypt31/32 in yeast) at later compartments [31,32], suggests its contribution to tethering is likely through promoting recruitment of Rab effector proteins, which could include some of the golgin tethers described above. 


\section{COG and GARP}

In contrast to TRAPP, COG and GARP contribute directly to vesicle recognition at the Golgi (Figure 2). COG is a complex comprised of eight subunits that is present on all Golgi cisternae and functions in intra-Golgi trafficking [33,34]. GARP, which is comprised of four subunits, is restricted to the TGN and functions in endosome to Golgi trafficking [35]. COG and GARP are members of the CATCHR family of MTCs, which are distantly related to each other and contain similar $\alpha$-helical bundled domains arranged in series [36]. This organization results in an extended conformation. Electron microscopy has shown that the COG and GARP complexes share a similar overall structure, both having flexible legs that extend from a central hub that is formed by the anti-parallel alignment of complex subunits $\left[37,38^{* *}, 39^{* *}\right]$. The extended conformation of COG and GARP raises the possibility that they could function as tethering complexes. Consistent with this notion, relocation of COG subunits to mitochondria was able to promote tethering of vesicles containing Golgi SNAREs [40]. However, this experiment was performed with over-expressed SNAREs, and so whether COG can tether native vesicles containing endogenous cargo remains to be demonstrated. Similarly, whether GARP is sufficient to tether vesicles has not been demonstrated.

Rather than functioning as vesicle tethers, that is in the initial vesicle capture event, we think it is more likely that COG and GARP act downstream of golgins to coordinate the transition from vesicle tethering to fusion (Figure 1). They are shorter than the golgins, which suggests they would be less efficient at vesicle capture over long distance. COG interacts with numerous trafficking components that include coat proteins, golgins, Rabs, SM proteins that regulate SNARE activity, and the SNAREs themselves 
[34]. GARP binding partners are less well defined but also include Rab and SNARE proteins $[35,41]$. Hence, COG and GARP are well placed to facilitate the transition from tethering to fusion. By interacting with components on both the vesicle and the target membrane, and by acting in conjunction with golgins, both the fidelity of vesicle recognition and the strength of vesicle attachment to the target membrane are likely greatly enhanced by COG and GARP. It is tempting to speculate that the elongated 'spidery' arms of the COG and GARP complexes bridge the various factors that act downstream of golgins to orchestrate the transition from one state to the next, ultimately leading to the pairing of cognate SNAREs on the vesicle and target membrane to drive membrane fusion. Both COG and GARP have been shown to promote SNARE pairing [42-44]. Structural analysis has indicated that the SNARE binding regions within COG and GARP are four helical bundles, reminiscent of that seen in SNARE complexes, suggesting COG and GARP could modulate SNARE complex assembly by swapping helices with those of the SNAREs, or by packing together with the SNARE helical regions, to help bring SNAREs together in a complex [37].

\section{COG sub-complexes}

Although we know much about COG interaction with binding partners and its architecture, how it works at a mechanistic level is poorly understood. Interestingly, despite being identified as a complex of eight subunits, recent evidence supports the notion that COG can also exist as two functionally relevant but physically distinct subcomplexes, comprising COG1-4 and COG5-8 [45**]. The COG1-4 sub-complex, which corresponds to lobe A of the entire COG complex, is enriched at the Golgi membrane whereas the COG5-8 sub-complex, corresponding to lobe B of the COG complex, is 
present on vesicular structures [45*]. This separation of COG sub-complexes implies they have distinct roles in the vesicle tethering process. In line with this idea, it has been shown in yeast that subunits Cog1-4, also referred to as the core complex, are essential, whereas Cog5-8 are dispensable for growth, at least under laboratory conditions $[37,46]$. Similarly, loss of lobe A subunits is lethal in Drosophila, whereas loss of lobe B subunits leads to milder phenotypes, and mutations in the COG1-4 subunits are more detrimental to humans than those in the COG5-8 subunits [33]. Hence, the COG1-4 sub-complex fulfills a vital function in vesicle traffic that cannot easily be bypassed, whereas that performed by the COG5-8 can, at least to some extent. Perhaps the interactions mediated by COG5-8 can be substituted for by other factors that the COG1-4 sub-complex can interact with. In the native wild-type situation, when all subunits are present, the separation of the two sub-complexes onto target membrane and vesicle suggests that a fully functional COG complex, generated by the association of the two sub-complexes, can only form upon vesicle tethering. This may be analogous to the assembly of the exocyst, a CATCHR complex involved in tethering of exocytic vesicles at the plasma membrane [36], and would allow COG to facilitate the transition to from tethering to fusion in the right place at the right time (Figure 1).

The GARP complex bears an interesting structural resemblance to the COG1-4 sub-complex [37,38**], suggesting that GARP and COG1-4 may function in a similar manner. GARP could therefore perform the same core functions that COG1-4 seems capable of, but for traffic at the TGN instead of within the Golgi stack. There does not appear to be an equivalent of the COG5-8 sub-complex for GARP, suggesting there are some mechanistic differences to COG. An alternative and more speculative possibility is 
that there is a GARP equivalent of the COG5-8 sub-complex, whose association with the core GARP complex is weaker than that between the COG sub-complexes. If it exists this sub-complex would be predicted to reside on vesicles tethered by GARP.

\section{SNARE pairing- the final specificity step}

It is well established that SNARE proteins mediate the docking and fusion of transport vesicles [47]. Although not the primary determinants of vesicle recognition, the pairing of cognate SNAREs still provides a layer of specificity to the vesicle recognition process that is downstream from the steps mediated by golgins and MTCs. The four-helix SNARE complex is formed through the pairing of one helix from a so-called R-SNARE, and three helices from so-called Q-SNAREs, typically with one of these SNAREs on the vesicle paring with two-three SNAREs on the target membrane [47]. For ER to Golgi trafficking vesicle-associated Bet1 pairs with syntaxin-5/GS27/ERS24 in the early Golgi [48]. For intra-Golgi traffic, GS15 on vesicles pairs with syntaxin-5/GS28/YKT6 within the Golgi stack [48]. At the TGN, vesicle associated VAMP3 or VAMP4 pairs with syntaxin-16/Vtila and either syntaxin-10 or syntaxin-6 respectively [48]. As mentioned above the COG and GARP complexes help promote SNARE pairing, acting in a selective way on the SNAREs involved in intra-Golgi and endosome-Golgi traffic [42-44], thus

ensuring that tethering is coupled with downstream docking and fusion of transport vesicles at the correct region of the Golgi apparatus (Figure 1).

\section{Unanswered questions and future directions}

What are the vesicle-associated determinants recognized by tethering motifs? 
Our understanding of the Golgi-associated vesicle tethering machinery has improved significantly over recent years. However, there are many unanswered questions. The recent identification of tethering motifs in the amino termini of golgins is an important discovery $\left[19^{* *}\right]$, but we do not know the determinants on the vesicle that they recognize. Such determinants may be protein or lipid. Vesicles are coated during their generation, but whether vesicle-associated coats contribute to tethering at the Golgi is unclear. GCC185 can bind to the AP1 component of the clathrin coat, suggesting possible involvement of the coat in tethering [49]. This interaction could act in conjunction with tethering by the extreme amino terminus of GCC185 to stabilize vesicle attachment, or it could contribute to the events that occur post-tethering [50]. Regardless, the interaction between GCC185 and AP1 implies that vesicles tethered by GCC185 must be at least partially coated. For other golgins the situation is less clear. Physical interaction with coat proteins has not been reported, and Golgi vesicles captured by several of the golgins when targeted to mitochondria appear to lack a coat [12**], suggesting that coats are not required for tethering in those cases, or that they are rapidly lost following tethering. Certainly in the case of tethering by the ALPS motif of GMAP-210, the coat must at least be partially uncoated prior to tethering to allow access to the vesicle lipids [15*]. Several subunits of COG bind to COPI [51-54]. Such interactions could contribute to vesicle recognition at the target membrane, acting in conjunction with other vesicle-associated factors to stabilize tethering and/or promote downstream events. However, another possibility is that the interaction with COPI acts further upstream to recruit COG into newly forming transport vesicles, as is likely to occur for the COG5-8 sub-complex 
$\left[45^{* *}\right]$. Further studies will be required to determine the precise role, if any, of the coat in vesicle recognition at the Golgi.

\section{Do all golgins tether vesicles?}

Interestingly, three of the golgins tested (giantin, CASP, and GCC185) failed to show activity in the mitochondrial tethering assay [12]. This could reflect the possibility that these golgins do not function as vesicle tethers at all, that they tether vesicles not present in the analysis. Another scenario could be that they tether non-vesicle membranes, which may include Golgi cisternae, or tubular regions of the Golgi such as the TGN, the fenestrated regions located between stacks within the Golgi ribbon, or tubular carriers that have been postulated to mediate intra-Golgi traffic [55]. Giantin is able to promote linking of Golgi stacks within the ribbon, suggesting a possible role in membrane tethering that is required to maintain Golgi organization as opposed to vesicle traffic [56]. It is also possible that tethering was not observed in the mitochondrial assay because the golgin tether is active only on the vesicle, as opposed to the target membrane, which would not have been detected the way the assay was designed [12**]. Certainly, some golgins can enter transport vesicles, but whether they are functional in this context is currently unclear. Most golgins are peripheral membrane proteins recruited to the Golgi membrane through interactions with small GTPases $[10,11]$. However, some golgins are membrane anchored (giantin, CASP, golgin-84), and so must be recycled within the Golgi stack to maintain their correct steady-state distribution. In this scenario it is likely that golgins are simply cargo that is tethering incompetent, possibly through adoption of a 'closed' inactive conformation, but it remains possible some, or all, may also work as 
vesicle-associated tethers. Further work will be required to discriminate between these possibilities.

\section{Mechanisms and additional factors?}

Vesicle recognition at the target membrane requires the coordinated action of golgins, MTCs, Rabs and SNAREs. These proteins act in concert to ensure that vesicle cargo is delivered to the correct Golgi region in a specific manner. The transition from initial capture to vesicle fusion is likely to require significant conformational rearrangements, but how these are brought about remains a mystery. Combining structural biology with more refined cell-based experiments, using both gain-of-function and loss-of-function assays, will undoubtedly provide important new insights. Such approaches will also help address issues such as whether golgins have 'open' and 'closed' conformations that correspond to active and inactive states respectively, which may be true for the tailanchored golgins that need to be delivered in vesicles to their site of action at the Golgi. It is also likely that additional Golgi tethering factors remain to be identified. Golgins share limited sequence identity, and so identifying new members of this family is not straightforward. Similarly, there may be additional MTCs that lack sequence similarity with the known tethering complexes, and we should also not exclude the possibility that other proteins not conforming to either golgin or MTC designations may remain to be discovered. Finally, whether golgins and MTCs can associate in tethering 'hotspots' is an interesting question. This has recently been shown for the Dsl MTC at the ER [57], and it tempting to speculate that analogous regions may also be present at the Golgi, providing a higher degree of spatial organization than currently appreciated. 


\section{References}

1. Nakamura N, Wei JH, Seemann J: Modular organization of the mammalian Golgi apparatus. Curr Opin Cell Biol 2012, 24:467-474.

2. Brandizzi F, Barlowe C: Organization of the ER-Golgi interface for membrane traffic control. Nat Rev Mol Cell Biol 2013, 14:382-392.

3. Gomez-Navarro N, Miller E: Protein sorting at the ER-Golgi interface. J Cell Biol 2016.

4. Stanley P: Golgi glycosylation. Cold Spring Harb Perspect Biol 2011, 3.

5. Papanikou E, Glick BS: Golgi compartmentation and identity. Curr Opin Cell Biol 2014, 29:74-81.

6. Papanikou E, Day KJ, Austin J, Glick BS: COPI selectively drives maturation of the early Golgi. Elife 2015, 4.

7. Mani S, Thattai M: Stacking the odds for Golgi cisternal maturation. Elife 2016, 5.

8. Guo Y, Sirkis DW, Schekman R: Protein sorting at the trans-Golgi network. Annu Rev Cell Dev Biol 2014, 30:169-206.

9. Progida C, Bakke O: Bidirectional traffic between the Golgi and the endosomes machineries and regulation. J Cell Sci 2016, 129:3971-3982.

10. Witkos TM, Lowe M: The Golgin Family of Coiled-Coil Tethering Proteins. Front Cell Dev Biol 2015, 3:86.

11. Gillingham AK, Munro S: Finding the Golgi: Golgin Coiled-Coil Proteins Show the Way. Trends Cell Biol 2016, 26:399-408.

12. Wong M, Munro S: Membrane trafficking. The specificity of vesicle traffic to the Golgi is encoded in the golgin coiled-coil proteins. Science 2014, 346:1256898. 
$[* *]$ A key paper showing that golgins are sufficient to tether transport vesicles and that golgin-mediated tethering occurs in a specific manner. The results indicate that golgins contribute to the specificity of trafficking at the Golgi apparatus.

13. Drin G, Morello V, Casella JF, Gounon P, Antonny B: Asymmetric tethering of flat and curved lipid membranes by a golgin. Science 2008, 320:670-673.

14. Sato K, Roboti P, Mironov AA, Lowe M: Coupling of vesicle tethering and Rab binding is required for in vivo functionality of the golgin GMAP-210. Mol Biol Cell 2015, 26:537-553.

[*] This paper shows that the ALPS motif and Rab2 binding site in GMAP-210 both contribute to functionality of the protein, with Rab2 binding likely operating downstream from the initial ALPS-mediated tethering event.

15. Magdeleine M, Gautier R, Gounon P, Barelli H, Vanni S, Antonny B: A filter at the entrance of the Golgi that selects vesicles according to size and bulk lipid composition. Elife 2016, 5.

$\left.{ }^{*}\right]$ In this paper the features of transport vesicles that are recognized by the ALPS motif of GMAP-210 are identifed. It is shown that the vesicle lipids and the packing of these lipids are recognized in a specific manner by the ALPS motif to impart selectivity to GMAP-210-mediated tethering.

16. Nakamura N, Lowe M, Levine TP, Rabouille C, Warren G: The vesicle docking protein p115 binds GM130, a cis-Golgi matrix protein, in a mitotically regulated manner. Cell 1997, 89:445-455. 
17. Seemann J, Jokitalo EJ, Warren G: The role of the tethering proteins p115 and GM130 in transport through the Golgi apparatus in vivo. Mol Biol Cell 2000, 11:635-645.

18. Marra P, Maffucci T, Daniele T, Tullio GD, Ikehara Y, Chan EK, Luini A, Beznoussenko G, Mironov A, De Matteis MA: The GM130 and GRASP65 Golgi proteins cycle through and define a subdomain of the intermediate compartment. Nat Cell Biol 2001, 3:1101-1113.

19. Wong M, Gillingham AK, Munro S: The golgin coiled-coil proteins capture different types of transport carriers via distinct $\mathbf{N}$-terminal motifs. BMC Biol 2017, 15:3. doi: 10.1186/s12915-016-0345-3.

[**] This paper identifies motifs in the amino terminal regions of golgins that mediate vesicle tethering. The motifs are specific such that golgins in different Golgi regions use different motifs for tethering of distinct vesicle types.

20. Cheung PY, Limouse C, Mabuchi H, Pfeffer SR: Protein flexibility is required for vesicle tethering at the Golgi. Elife 2015, 4.

[**] This study shows that the golgin GCC185 can adopt a shorter bent conformation due to its inherent structural flexibility. This flexibility is important for GCC185 function at the Golgi. It is also shown that the extreme amino terminus of GCC185 can adopt a splayed conformation to capture vesicles.

21. Sinka R, Gillingham AK, Kondylis V, Munro S: Golgi coiled-coil proteins contain multiple binding sites for Rab family G proteins. J Cell Biol 2008, 183:607615. 
22. Munro S: The golgin coiled-coil proteins of the Golgi apparatus. Cold Spring Harb Perspect Biol 2011, 3.

23. Murray DH, Jahnel M, Lauer J, Avellaneda MJ, Brouilly N, Cezanne A, MoralesNavarrete H, Perini ED, Ferguson C, Lupas AN, et al.: An endosomal tether undergoes an entropic collapse to bring vesicles together. Nature 2016, 537:107-111.

$[*]$ It is shown that the endosomal coiled-coil tethering protein EEA1 undergoes collapse upon binding to active Rab5, shortening the length of the protein that is important for mediating the transition from endosome tethering to fusion.

24. Ishida R, Yamamoto A, Nakayama K, Sohda M, Misumi Y, Yasunaga T, Nakamura N: GM130 is a parallel tetramer with a flexible rod-like structure and Nterminally open (Y-shaped) and closed (I-shaped) conformations. FEBS J 2015, 282:2232-2244.

25. Whyte JR, Munro S: Vesicle tethering complexes in membrane traffic. J Cell Sci 2002, 115:2627-2637.

26. Brunet S, Sacher M: In sickness and in health: the role of TRAPP and associated proteins in disease. Traffic 2014, 15:803-818.

27. Kim JJ, Lipatova Z, Segev N: TRAPP Complexes in Secretion and Autophagy. Front Cell Dev Biol 2016, 4:20.

28. Wang W, Sacher M, Ferro-Novick S: TRAPP stimulates guanine nucleotide exchange on Ypt1p. J Cell Biol 2000, 151:289-296.

29. Jones S, Newman C, Liu F, Segev N: The TRAPP complex is a nucleotide exchanger for Ypt1 and Ypt31/32. Mol Biol Cell 2000, 11:4403-4411. 
30. Yamasaki A, Menon S, Yu S, Barrowman J, Meerloo T, Oorschot V, Klumperman J, Satoh A, Ferro-Novick S: mTrs130 is a component of a mammalian TRAPPII complex, a Rab1 GEF that binds to COPI-coated vesicles. Mol Biol Cell 2009, 20:4205-4215.

31. Morozova N, Liang Y, Tokarev AA, Chen SH, Cox R, Andrejic J, Lipatova Z, Sciorra VA, Emr SD, Segev N: TRAPPII subunits are required for the specificity switch of a Ypt-Rab GEF. Nat Cell Biol 2006, 8:1263-1269.

32. Thomas LL, Fromme JC: GTPase cross talk regulates TRAPPII activation of Rab11 homologues during vesicle biogenesis. J Cell Biol 2016, 215:499-513.

33. Miller VJ, Ungar D: Re'COG'nition at the Golgi. Traffic 2012, 13:891-897.

34. Willett R, Ungar D, Lupashin V: The Golgi puppet master: COG complex at center stage of membrane trafficking interactions. Histochem Cell Biol 2013, 140:271-283.

35. Bonifacino JS, Hierro A: Transport according to GARP: receiving retrograde cargo at the trans-Golgi network. Trends Cell Biol 2011, 21:159-167.

36. Yu IM, Hughson FM: Tethering factors as organizers of intracellular vesicular traffic. Annu Rev Cell Dev Biol 2010, 26:137-156.

37. Lees JA, Yip CK, Walz T, Hughson FM: Molecular organization of the COG vesicle tethering complex. Nat Struct Mol Biol 2010, 17:1292-1297.

38. Chou HT, Dukovski D, Chambers MG, Reinisch KM, Walz T: CATCHR, HOPS and CORVET tethering complexes share a similar architecture. Nat Struct Mol Biol 2016, 23:761-763.

[**] This study shows that the structures of the Golgi-localized COG1-4 sub-complex 
and GARP complex and endosomal HOPS and CORVET complexes share a

conserved structure, as determined by electron microscopy. They have an

elongated spidery shape with flexible legs protruding from a central hub. See also

reference 39.

39. Ha JY, Chou HT, Ungar D, Yip CK, Walz T, Hughson FM: Molecular architecture of the complete COG tethering complex. Nat Struct Mol Biol 2016, 23:758-760. $[* *]$ This study describes the structure of the entire octameric COG complex, as revealed by electron microscopy. It has 4-5 flexible legs that result in an overall extended conformation. See also reference 38 .

40. Willett R, Kudlyk T, Pokrovskaya I, Schonherr R, Ungar D, Duden R, Lupashin V: COG complexes form spatial landmarks for distinct SNARE complexes. Nat Commun 2013, 4:1553.

41. Gershlick DC, Schindler C, Chen Y, Bonifacino JS: TSSC1 is novel component of the endosomal retrieval machinery. Mol Biol Cell 2016, 27:2867-2878.

42. Shestakova A, Suvorova E, Pavliv O, Khaidakova G, Lupashin V: Interaction of the conserved oligomeric Golgi complex with t-SNARE Syntaxin5a/Sed5 enhances intra-Golgi SNARE complex stability. J Cell Biol 2007, 179:11791192.

43. Perez-Victoria FJ, Bonifacino JS: Dual roles of the mammalian GARP complex in tethering and SNARE complex assembly at the trans-golgi network. Mol Cell Biol 2009, 29:5251-5263. 
44. Laufman O, Hong W, Lev S: The COG complex interacts with multiple Golgi SNAREs and enhances fusogenic assembly of SNARE complexes. J Cell Sci 2013, 126:1506-1516.

45. Willett R, Blackburn JB, Climer L, Pokrovskaya I, Kudlyk T, Wang W, Lupashin V: COG lobe B sub-complex engages v-SNARE GS15 and functions via regulated interaction with lobe A sub-complex. Sci Rep 2016, 6:29139.

[**] This study shows that COG can exist as two distinct membrane-associated subcomplexes that reside either on the target Golgi membrane (COG1-4, lobe A) or the transport vesicle (COG5-8, lobe B), respectively. It suggests a mechanism for linking tethering with full assembly and functionality of the COG complex.

46. Whyte JR, Munro S: The Sec34/35 Golgi transport complex is related to the exocyst, defining a family of complexes involved in multiple steps of membrane traffic. Dev Cell 2001, 1:527-537.

47. Jahn R, Scheller RH: SNAREs--engines for membrane fusion. Nat Rev Mol Cell Biol 2006, 7:631-643.

48. Malsam J, Sollner TH: Organization of SNAREs within the Golgi stack. Cold Spring Harb Perspect Biol 2011, 3:a005249.

49. Brown FC, Schindelhaim CH, Pfeffer SR: GCC185 plays independent roles in Golgi structure maintenance and AP-1-mediated vesicle tethering. J Cell Biol 2011, 194:779-787.

50. Cheung PY, Pfeffer SR: Transport Vesicle Tethering at the Trans Golgi Network: Coiled Coil Proteins in Action. Front Cell Dev Biol 2016, 4:18. 
51. Ram RJ, Li B, Kaiser CA: Identification of Sec36p, Sec37p, and Sec38p: components of yeast complex that contains Sec34p and Sec35p. Mol Biol Cell 2002, 13:1484-1500.

52. Suvorova ES, Duden R, Lupashin VV: The Sec34/Sec35p complex, a Ypt1p effector required for retrograde intra-Golgi trafficking, interacts with Golgi SNAREs and COPI vesicle coat proteins. $J$ Cell Biol 2002, 157:631-643.

53. Zolov SN, Lupashin VV: Cog3p depletion blocks vesicle-mediated Golgi retrograde trafficking in HeLa cells. $J$ Cell Biol 2005, 168:747-759.

54. Miller VJ, Sharma P, Kudlyk TA, Frost L, Rofe AP, Watson IJ, Duden R, Lowe M, Lupashin VV, Ungar D: Molecular insights into vesicle tethering at the Golgi by the conserved oligomeric Golgi (COG) complex and the golgin TATA element modulatory factor (TMF). J Biol Chem 2013, 288:4229-4240.

55. Trucco A, Polishchuk RS, Martella O, Di Pentima A, Fusella A, Di Giandomenico D, San Pietro E, Beznoussenko GV, Polishchuk EV, Baldassarre M, et al.: Secretory traffic triggers the formation of tubular continuities across Golgi subcompartments. Nat Cell Biol 2004, 6:1071-1081.

56. Koreishi M, Gniadek TJ, Yu S, Masuda J, Honjo Y, Satoh A: The golgin tether giantin regulates the secretory pathway by controlling stack organization within Golgi apparatus. PLoS One 2013, 8:e59821.

57. Schroter S, Beckmann S, Schmitt HD: ER arrival sites for COPI vesicles localize to hotspots of membrane trafficking. EMBO J 2016, 35:1935-1955. 


\section{Acknowledgements}

We would like to thank Drs Joachim Seemann (UT Southwestern Medical Center, Dallas, USA) and Daniel Ungar (University of York, UK) for critical reading of the manuscript. Work in the Lowe lab is funded by the BBSRC, MRC, Wellcome Trust and the Lowe Syndrome Trust.

\section{Figure Legends}

Figure 1. Model for how golgin, MTC and SNARE proteins combine to ensure specific recognition and fusion of a Golgi transport vesicle.

Formation of an intra-Golgi transport vesicle is mediated by COPI coat assembly. Certain components required for vesicle recognition later on (Rabs, SNAREs, COG complex lobe B) are incorporated into the vesicle at this stage. Following budding, a vesicle-associated determinant is recognized by the amino terminal tethering motif of a golgin. In the case of GMAP-210, loss of the coat must occur to allow access of the ALPS motif to the lipids on the vesicle surface to which it binds. The vesicle-associated determinants recognized by amino terminal motifs of other golgins are unknown. Post-capture, the vesicle is moved closer to the target membrane through conformational changes of the golgin, which may involve binding to Rab GTPases. Additional stabilization of the tethered vesicle is achieved through interactions of the COG multi-subunit tethering complex (MTC). The vesicle-associated lobe B sub-complex of COG pairs with the Golgi- 
membrane-localized lobe A to form a stable attachment. These interactions also promote SNARE pairing, which leads to the conversion from vesicle tethering to fusion.

\section{Figure 2. Vesicle recognition machinery at the Golgi apparatus.}

Different golgins and multi-subunit tethering complexes, which combine to provide specific recognition of transport vesicles, are localized to distinct Golgi regions. The golgins are long, coiled-coil proteins that extend from the Golgi surface and capture vesicles by membrane-distal amino $(\mathrm{N})$-terminal tethering motifs. They differ in localization and tethering specificity, with golgins at the cis-Golgi capturing ER-and ERGIC-derived vesicles, golgins localized to cisternal rims capturing intra-Golgi transport vesicles, and trans-Golgi golgins capturing endosome-derived carriers. The COG complex is involved in intra-Golgi transport and consists of two lobes: lobe A (Cog1-4 subunits) predominantly associated with Golgi membranes, and lobe B (Cog5-8 subunits), which is found on vesicles. Interaction of both lobes and formation of the complete COG complex is predicted to occur during or shortly after golgin-mediated tethering. The GARP complex is composed of four subunits (Vps51-54) and contributes to tethering of vesicles at the trans-Golgi, but the mechanisms remain poorly defined. 


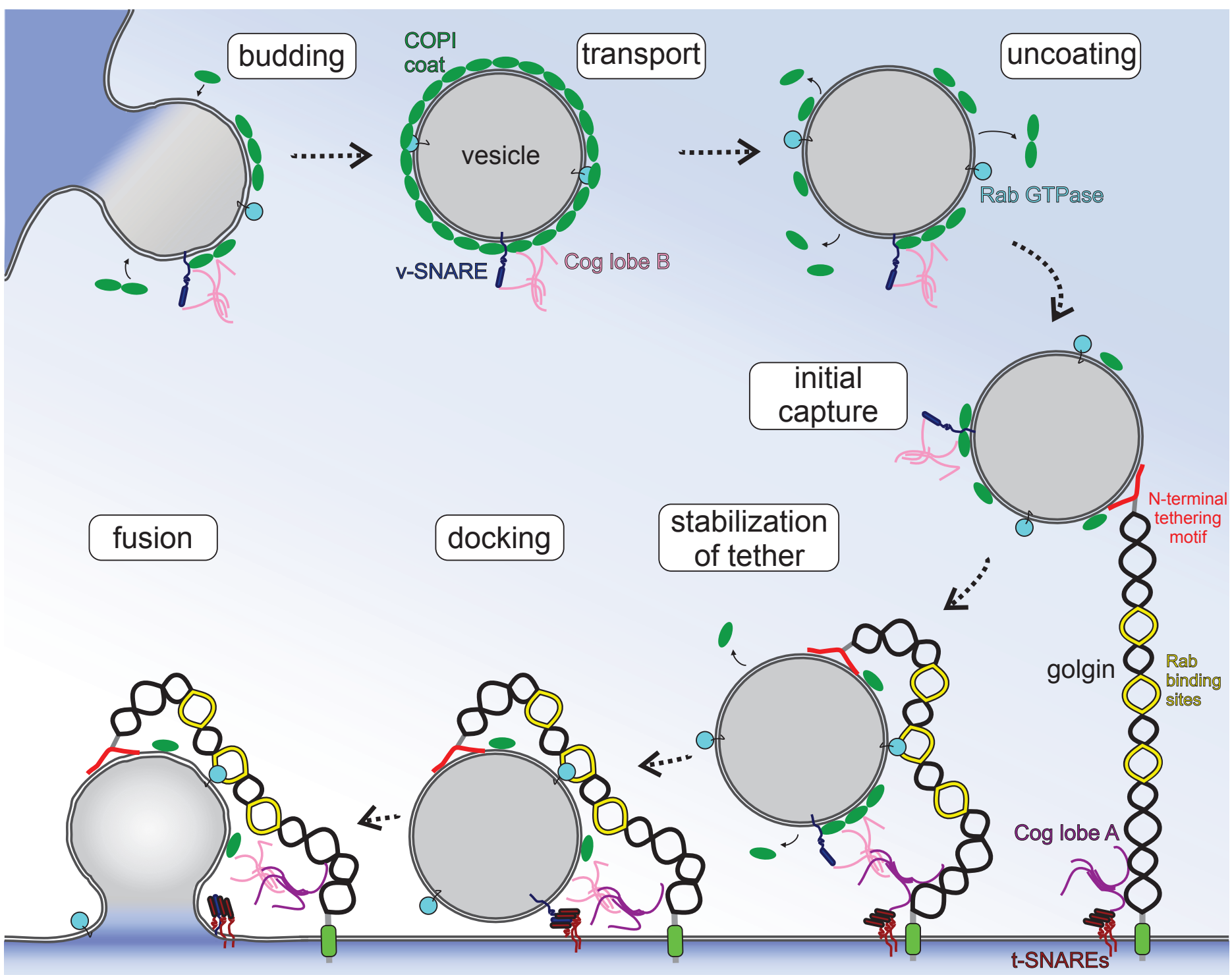



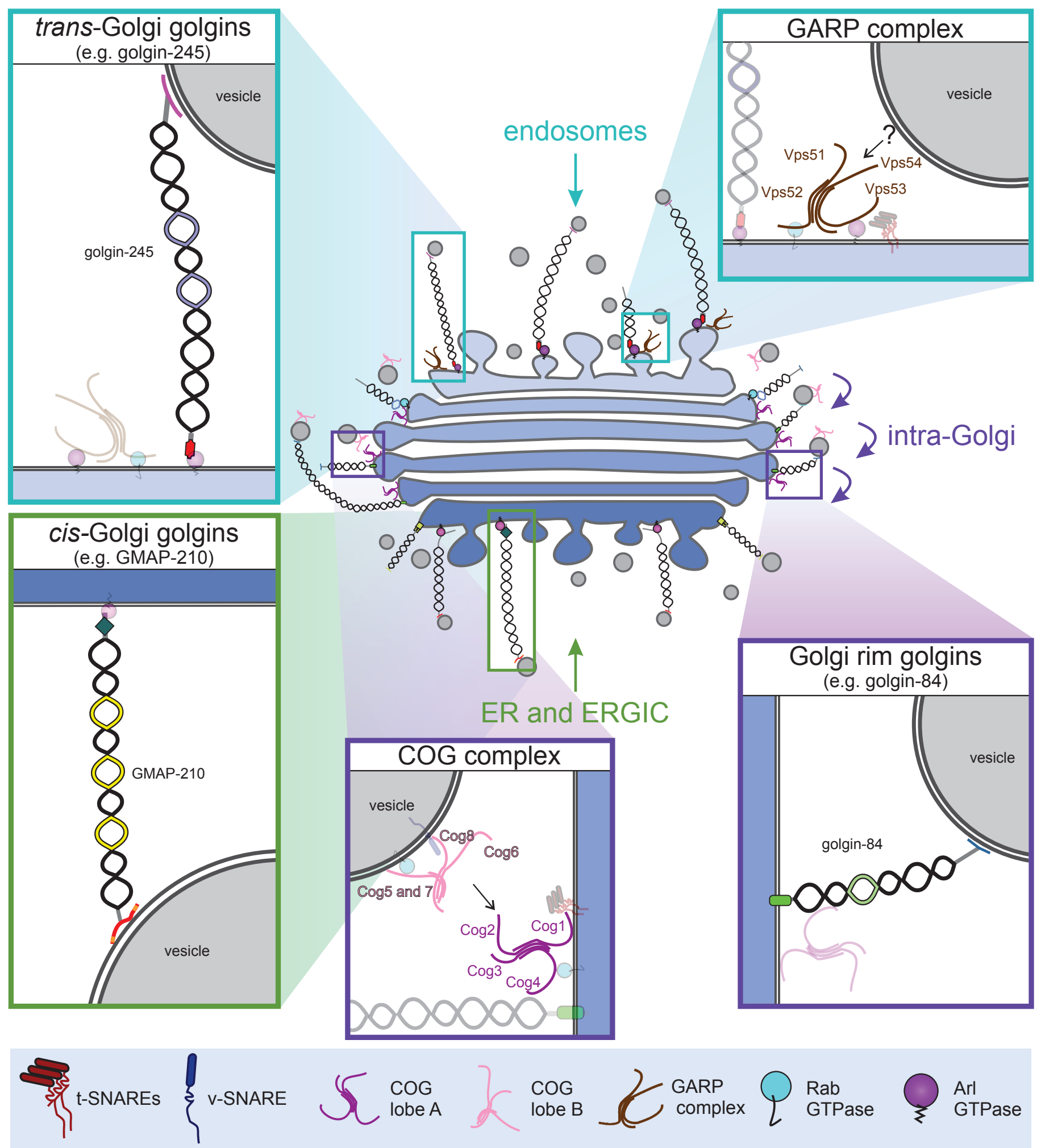


\section{Highlights:}

- Coiled-coil golgin proteins capture transport vesicles in a specific manner

- Golgins contain vesicle tethering motifs

- Multi-subunit tethering complexes (MTCs) likely act downstream of golgins to orchestrate vesicle tethering

- Golgi MTCs share a similar structural organization with elongated 'spidery' arms 